\title{
ESTUDOS DE VIABILIDADE DE SEMENTES DE CANDEIA Eremanthus erythropappus (DC.) MacLeish POR MEIO DE TESTES DE GERMINAÇÃO E RAIOS X
}

\author{
Selma dos Santos Feitosa ${ }^{1}$, Antonio Cláudio Davide ${ }^{2}$, Olívia Alvina Oliveira Tonetti ${ }^{3}$, \\ Juliano Ricardo Fabricante ${ }^{4}$, Jandislau José Lui ${ }^{5}$

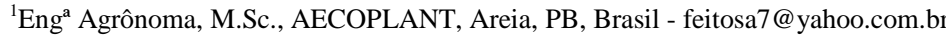 \\ ${ }^{2}$ Eng. Agrônomo, Dr., Depto. de Ciências Florestais, UFLA, Lavras, MG, Brasil - acdavide@ufla.br \\ ${ }^{3}$ Eng $^{a}$ Florestal, M.Sc., Depto. de Ciências Florestais, UFLA, Lavras, MG, Brasil - oaotonetti@ yahoo.com.br \\ ${ }^{4}$ Biólogo, Doutorando em Agronomia, UFPB, Areia, PB, Brasil - julianofabricante@ hotmail.com \\ ${ }^{5}$ Eng. Agrônomo, Dr., Faculdade de Agronomia, UFT, Gurupi, TO, Brasil - jandislaului@ hotmail.com
}

Recebido para publicação: 05/06/2008 - Aceito para publicação: 11/08/2008

\begin{abstract}
Resumo
A candeia, Eremanthus erythropappus (DC.), é uma espécie florestal com múltiplos usos, como o do seu óleo, que possui propriedades antiflogísticas, dermatológicas e espasmódicas. Por possuir sementes com baixa porcentagem de germinação, o objetivo deste trabalho foi estudar a qualidade dessas sementes, visando conhecer melhor o seu desempenho germinativo. Realizou-se no Laboratório de Sementes Florestais da Universidade Federal de Lavras - UFLA, um experimento utilizando-se sementes originárias de Carrancas, Itabirito, Morro do Pilar, Baependi e Delfim Moreira, aplicando-se o delineamento experimental inteiramente casualizado com quatro repetições de 25 sementes. Realizou-se teste de germinação com temperaturas alternadas de $20-30{ }^{\circ} \mathrm{C}$ e fotoperíodos de 10 e 14 horas de luz, ocorrendo a avaliação no $14^{\circ}$ dia. As sementes não germinadas foram dissecadas para verificação da presença ou ausência do embrião, sendo divididas em sementes cheias e vazias. Sementes das mesmas progênies submetidas ao teste de germinação passaram por análise de raios X, com a finalidade de melhor visualização das estruturas internas, ou seja, verificar a presença ou não de embrião. Foram selecionadas duas progênies de cada procedência que se destacaram nos testes anteriores (germinação e raios X). Essas sementes passaram por soprador e a fração retida foi submetida à análise de raios X e germinação. Para as características avaliadas, as progênies C6, I18, M24, B9 e D2 se destacaram em qualidade, conforme a germinação e semente vazia. As sementes das progênies C25, I17, M22, B24 e D16 foram as que apresentaram as taxas mais baixas, respectivamente. Após o tratamento com soprador, constatou-se que as progênies atingiram um alto número de sementes cheias, possibilitando um incremento na germinação de $9 \%$ para I12 e de $47 \%$ para B24. O baixo percentual germinativo pode ter se dado em razão do grande número de sementes sem embrião.

Palavras-chave: Soprador de semente; espécie florestal; Minas Gerais.
\end{abstract}

\begin{abstract}
Viability study of seeds of candeia Eremanthus erythropappus (DC.) MacLeish by germination and $x$ ray tests. Eremanthus erythropappus (DC.) is a forest species, with multiples as oil extraction activity. As they have a seed with low percentage of germination, the aim of this study was to improve seeds quality of candeia. So, it had been carried out an experiment, using seeds from Carrancas, Itabirito, Morro do Pilar, Baependi and Delfim Moreira, using a randomized block with four repetitions. Germination test was carried out with temperatures of $20^{\circ}-30^{\circ} \mathrm{C} / 10$ and 14 hours light, being evaluated at $14^{\text {th }}$ days, non-germinated seeds were dried to check the presence or not and embryo state, being divided in firm, dead and empty seeds. Seeds of the same progenies by germination test were subjected to x-ray analysis. It had been selected two progenies of each tests (germination and $\mathrm{x}$-ray); these seeds were winded and portion was subjected to x-ray and germination tests. To the evaluated features, progenies C6, I18, M24, B9 and D2 had better quality, according to germination and empty seed. Progenies C25, I17, M22, B24 and D16 were treatments which shwed the lowest means, respectively. After treatments with wind, it had been noted progenies reached a higher number of firm seeds, giving an increment on germination of $9 \%$ to I12 and $47 \%$ to B24. A low germinative percentage can be reasonable by a high number of empty aquenious.

Keywords: Seed blower; forest species; Minas Gerais; empty seed.
\end{abstract}




\section{INTRODUÇÃO}

A candeia, Eremanthus erythropappus (DC.), ocorre desde o estado da Bahia até o Rio Grande do Sul (CARVALHO, 1994; TEIXEIRA et al., 1996). É uma espécie arbórea de grande importância econômica e que está sendo o centro de atenção de pequenos e médios agricultores em diversas regiões do estado de Minas Gerais, tendo em vista o principal produto de interesse dessa espécie, o óleo essencial extraído de toda a planta que possui propriedades farmacológicas, destacando-se pelo seu alto valor comercial. A candeia pertence ao grupo ecológico das pioneiras frequentemente encontradas desde o estado da Bahia até o estado do Rio de Janeiro (NEMAF, 2003), formando grandes populações que podem dominar ambientes de cerrado e campo rupestre. Ocorre também em florestas com algum grau de perturbação, pois é uma espécie heliófila e a entrada de luz a beneficia (CARVALHO, 1994; MENDONÇA; LINS 2000).

Sua ocorrência é típica de locais montanhosos, pedregosos, com solos de baixa fertilidade. Esse fator, aliado a sua alta capacidade reprodutiva e baixa exigência nutricional, facilita o seu cultivo e manejo (SCOLFORO et al., 2002). Enfim, a candeia é adaptada a locais em que seria difícil a implantação de culturas agrícolas ou mesmo de outras espécies florestais.

A utilização da E. erythropappus pode ser um diferencial para o produtor rural que, além de recuperar o solo com uma cobertura vegetal, agregando matéria orgânica e melhorando sua estrutura, pode, por meio de um plano de manejo adequado, beneficiar-se da madeira, de amplo uso para moirões e extração de óleo, cujo princípio ativo, o alfabisabolol, possui propriedades antiflogísticas, antibacterianas, antimicóticas, dermatológicas e espasmódicas, sendo atualmente utilizado na fabricação de medicamentos e cosméticos (PÉREZ, 2001; SALUSTIANO et al., 2006).

Dada a importância econômica de E. erythropappus, tem ocorrido aumento na sua exploração, levando a novos plantios, fato que tem proporcionado aumento na demanda por sementes de melhor qualidade. No entanto, trabalhos iniciais mostram que o percentual germinativo das sementes de algumas espécies de Eremanthus é baixo (DAVIDE et al., 2003; VELTEN; GARCIA, 2005; TONETTI et al., 2006), sendo sua causa ainda desconhecida. Uma hipótese levantada por Chaves e Ramalho (1996) é que seja devido a um grande número de sementes vazias, ou seja, que não continham embrião.

Conforme Carvalho et al. (2000), o ciclo de vida das espécies é influenciado diretamente por condições climáticas, sendo o regime hídrico o fator fundamental na qualidade da semente produzida. Para isso, é necessário que haja no solo água disponível para a planta, do contrário, formam-se sementes chochas (CARVALHO et al., 2000). Outro fator determinante na qualidade das sementes é o estado fisiológico no momento em que são colhidas, pois, quando imaturas, apresentam baixa germinação (GOMES et al., 2002).

Uma das maneiras possíveis para a avaliação desse fator sobre as sementes é a sua análise física pela técnica de raios X, caracterizada pela avaliação das estruturas internas do embrião (SEED NEWS, 2001). Diferenciando-as em vazias, cheias e com danos, as informações podem auxiliar na identificação da qualidade física e fisiológica das sementes, que acabam refletindo diretamente nos índices de germinação da espécie (TONETTI et al., 2006).

Em geral, para a melhoria da pureza física, da germinação e do vigor das sementes, utilizam-se máquinas de ar e peneiras para remover todas as impurezas e sementes indesejáveis do produto colhido (FONSECA et al., 2003; FONSECA et al., 2004).

O conhecimento sobre o comportamento germinativo das sementes de espécies nativas é fundamental para a reabilitação de áreas degradadas e constitui providência essencial para protegê-las da ameaça de extinção. Há, porém, carência de informações sobre essas espécies (CARPI et al., 1996). Desse modo, tornam-se fundamentais os estudos para a utilização e exploração de forma racional.

Em função da importância ecológica e comercial que a candeia apresenta, esta pesquisa teve como objetivo avaliar, por meio de análise radiográfica e teste de germinação, a viabilidade de suas sementes provenientes de diferentes progênies colhidas no estado de Minas Gerais.

\section{MATERIAL E MÉTODOS}

O presente estudo foi realizado no Laboratório de Sementes Florestais do Departamento de Ciências Florestais da Universidade Federal de Lavras (UFLA). Foram utilizadas sementes provenientes de cinco municípios do estado de Minas Gerais: Carrancas (C), Baependi (B), Delfim Moreira (D), 
Itabirito (I) e Morro do Pilar (M). As sementes foram mantidas em câmara fria (60\% de umidade relativa a $5{ }^{\circ} \mathrm{C}$ ), em sacos plásticos semipermeáveis até o início dos testes. Foram consideradas como sementes as unidades de dispersão conhecidas por aquênio.

A partir do conhecimento do histórico da germinação das sementes de cada procedência, foram selecionadas seis progênies, sendo três com melhor desempenho germinativo (C6, C8, C1, B2, B8, B9, D3, D1, D2, I9, I18, I3, M24, M3 e M1), e três com pior desempenho germinativo (C2, C25, C4, B19, B20 B24, D17, D16, D19, I11, I12, I17, M22, M5 e M18) de cada local.

Após a seleção efetuada, procedeu-se a um teste de germinação, em delineamento inteiramente casualizado (DIC) com quatro repetições de 25 sementes, distribuídas em placas de Petri sobre duas folhas de papel filtro, e mantidas em germinador iluminado com lâmpadas fluorescentes $(20 \mathrm{~W})$, por um período de 10 horas a $30^{\circ} \mathrm{C}$, alternado por 14 horas de escuro a $20^{\circ} \mathrm{C}$ e durante 14 dias. As sementes não germinadas foram dissecadas para verificação da presença ou ausência do embrião. Em seguida, foram divididas em sementes cheias e vazias, sendo o resultado expresso em porcentagem média de plântulas normais, conforme protocolo indicado por Tonetti (2004).

Para a análise por meio de raios $\mathrm{X}$, foram utilizadas quatro repetições de 25 sementes de cada progênie avaliada, as quais foram coladas sobre fita adesiva de dupla face em placas de acrílico apresentando $2 \mathrm{~mm}$ de espessura, medindo $16 \mathrm{~cm}$ de largura e $17 \mathrm{~cm}$ comprimento. Utilizou-se o aparelho de raios X marca Faxitron $\odot$, modelo $43855 \mathrm{X}$, na potência de $30 \mathrm{Kv}$ por 45 segundos e filme radiográfico para obtenção das imagens.

De acordo com a morfologia interna visualizada nas radiografias, as sementes foram divididas em duas categorias: cheias (aquelas com embrião bem formado, ocupando todo o interior do aquênio) e vazias (aquelas com ausência de embrião).

Conforme os resultados obtidos no teste de germinação e com base nas imagens radiográficas, foram selecionadas duas progênies de cada procedência das sementes. Utilizou-se como critério de seleção os melhores e piores resultados de cada avaliação efetuada, ou seja, formaram-se dois lotes de sementes, um com maior porcentagem de germinação e maior número de sementes cheias, e outro com menor percentual de germinação e menor número de sementes cheias, pela análise de raios X.

As sementes foram submetidas a separação por soprador tipo Seed Blower, regulado na abertura 6.0 e tempo de ventilação de 30 segundos (TONETTI, 2004), visando a melhoria da qualidade dessas sementes, sendo novamente submetidas a análise de raios $\mathrm{X}$ e teste de germinação.

Os dados obtidos foram analisados pelo programa estatístico SAEG com probabilidade de $\mathrm{F}<1 \%$, e as médias foram comparadas pelo teste de Tukey a 0,05 .

\section{RESULTADOS E DISCUSSÃO}

Foram encontradas diferenças significativas entre as progênies de uma mesma procedência quanto às características de germinação, sementes vazias e cheias, por meio da análise de variância e posterior aplicação do teste de médias (Tukey a $5 \%$ de probabilidade).

Para as características de germinação e sementes vazias, observou-se que as progênies C6, I18, M24, B9 e D2 se destacaram em qualidade, sugerindo-se que seja em razão do menor número de sementes sem a presença de embrião, podendo também estar relacionado esse fato a características genéticas da própria progênie e fatores ambientais de cada procedência. Em relação a essas características, as progênies que apresentaram maior número de sementes vazias e menor percentual de germinação foram C25, I12, M22, B24 e D16.

Com base nas análises radiográficas, as progênies que se sobressaíram, revelando um maior número de sementes cheias, foram C6, I9, M3, B2 e D2, sendo que as progênies M3 e B2 não se destacaram como as melhores do teste de germinação, provavelmente porque as sementes cheias se encontravam no estádio mais avançado de desenvolvimento do embrião, em relação às sementes com embriões malformados ou ausência deles, evidenciando uma causa natural da espécie estudada.

As tabelas 1 e 2 trazem os resultados do teste de germinação e de sementes vazias verificados pela dissecação das sementes não germinadas, após o teste, e o percentual de sementes cheias pela análise de raios $X$.

Essa causa pode estar associada à falta de uniformidade no florescimento, evento comum entre as espécies florestais, o que resulta em embriões com diferentes estágios de desenvolvimento, fato ressaltado por Machado; Cícero (2003), em estudo sobre morfologia de sementes de Lithraea molleoides. 
Quando as sementes foram submetidas ao soprador, constatou-se que, exceto as progênies I12 e B24, todas as demais apresentaram um alto número de sementes cheias, após passarem pelo soprador de fluxo de ar e serem analisadas através de raios X. Isso mostra que a passagem pelo soprador foi eficiente, aumentando a pureza física das sementes ao eliminar as sementes vazias dos lotes originais. Mostra também que as sementes com maior densidade foram mais nutridas, superando aquelas formadas mais tardiamente. Souza et al. (2001) afirmam que sementes com maior densidade mostraram melhores resultados de viabilidade e vigor nos testes realizados. Apesar dessas duas progênies não terem atingido um alto número de sementes cheias (conforme verificado na radiografia), pode-se observar que ainda assim houve melhoria nesses casos, como pode ser observado nas tabelas 1 e 2 , que possibilitou um incremento de $9 \%$ de sementes cheias para a progênie I12. Já para a progênie B24, o aumento foi ainda mais significativo, aumentando em $47 \%$ as sementes cheias na amostra.

Tabela 1. Percentual de germinação e de sementes vazias obtido pelo teste de germinação e percentual de sementes cheias obtido pelo teste de raios $\mathrm{X}$, provenientes de cinco municípios do estado de Minas Gerais (MG).

Table 1. Porcentual of germination and seed empty obtained by the test of seed germination percentage and floods obtained by x-ray test, from five municipalities in the state of Minas Gerais.

\begin{tabular}{|c|c|c|c|c|}
\hline Procedência & Progênie & Germinação (\%) & Sementes vazias (\%) & $\begin{array}{c}\text { Sementes cheias } \\
(\%)(\text { raios X) }\end{array}$ \\
\hline \multirow[t]{6}{*}{ Baependi } & B9 & $36 \mathrm{a}$ & $63 a$ & $26 a$ \\
\hline & B2 & $23 \mathrm{ab}$ & $77 \mathrm{a}$ & $32 \mathrm{a}$ \\
\hline & B8 & $15 \mathrm{bc}$ & $85 \mathrm{ab}$ & $23 a b$ \\
\hline & B20 & $9 \mathrm{c}$ & $90 \mathrm{ab}$ & $14 \mathrm{ab}$ \\
\hline & B19 & $8 \mathrm{c}$ & $91 b$ & $32 a$ \\
\hline & B24 & $5 \mathrm{c}$ & $94 \mathrm{~b}$ & $2 b$ \\
\hline \multirow[t]{6}{*}{ Carrancas } & C6 & $40 \mathrm{a}$ & $60 \mathrm{a}$ & $36 \mathrm{a}$ \\
\hline & $\mathrm{C} 4$ & $32 \mathrm{ab}$ & $67 \mathrm{ab}$ & $21 \mathrm{ab}$ \\
\hline & $\mathrm{C} 1$ & $21 \mathrm{abc}$ & $78 a b c$ & $21 \mathrm{ab}$ \\
\hline & $\mathrm{C} 8$ & $20 a b c$ & $79 \mathrm{abc}$ & $32 \mathrm{~b}$ \\
\hline & $\mathrm{C} 2$ & $12 b c$ & $87 \mathrm{bc}$ & $7 \mathrm{~b}$ \\
\hline & $\mathrm{C} 25$ & $6 \mathrm{c}$ & $93 \mathrm{c}$ & $5 \mathrm{~b}$ \\
\hline \multirow[t]{6}{*}{ Delfim Moreira } & D2 & $19 \mathrm{a}$ & $74 a$ & $21 \mathrm{a}$ \\
\hline & D1 & $11 \mathrm{ab}$ & $89 \mathrm{ab}$ & $13 \mathrm{ab}$ \\
\hline & D3 & $6 \mathrm{~b}$ & $82 \mathrm{ab}$ & $9 \mathrm{ab}$ \\
\hline & D19 & $4 b$ & $95 \mathrm{~b}$ & $4 \mathrm{~b}$ \\
\hline & D17 & $0 \mathrm{~b}$ & $96 \mathrm{~b}$ & $3 \mathrm{~b}$ \\
\hline & D16 & $0 \mathrm{~b}$ & $99 \mathrm{~b}$ & $5 \mathrm{~b}$ \\
\hline \multirow[t]{6}{*}{ Itabirito } & I18 & $25 \mathrm{a}$ & $72 \mathrm{a}$ & $12 \mathrm{a}$ \\
\hline & I9 & $13 \mathrm{ab}$ & $86 \mathrm{ab}$ & $16 \mathrm{a}$ \\
\hline & I3 & $11 \mathrm{ab}$ & $88 \mathrm{ab}$ & $7 \mathrm{a}$ \\
\hline & I11 & $3 \mathrm{~b}$ & $94 \mathrm{~b}$ & $2 \mathrm{a}$ \\
\hline & $\mathrm{I} 17$ & $3 \mathrm{~b}$ & $97 \mathrm{~b}$ & $6 \mathrm{a}$ \\
\hline & I12 & $2 \mathrm{~b}$ & $92 \mathrm{~b}$ & $5 \mathrm{a}$ \\
\hline \multirow[t]{6}{*}{ Morro do Pilar } & M24 & $39 a$ & $59 a$ & $27 \mathrm{ab}$ \\
\hline & M3 & $14 \mathrm{~b}$ & $84 \mathrm{~b}$ & $37 \mathrm{~b}$ \\
\hline & M1 & $14 \mathrm{~b}$ & $86 \mathrm{~b}$ & $7 \mathrm{c}$ \\
\hline & M5 & $14 \mathrm{~b}$ & $86 \mathrm{~b}$ & $16 \mathrm{bc}$ \\
\hline & M18 & $12 \mathrm{~b}$ & $86 \mathrm{~b}$ & $11 \mathrm{bc}$ \\
\hline & M22 & $4 \mathrm{~b}$ & $96 \mathrm{~b}$ & $6 \mathrm{c}$ \\
\hline
\end{tabular}

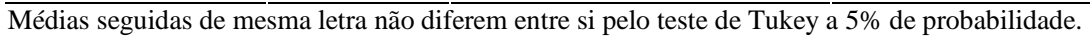

Os resultados do teste de germinação mostraram que a progênie I12 atingiu o menor percentual de germinação. Ainda assim houve melhoria na sua qualidade, com aumento de $10 \%$. Para as progênies M24 e M22, que apresentavam 39\% e 4\%, respectivamente, de germinação, após a separação obtiveram 
valores com diferença significativa, com aumento de $45 \%$ e $82 \%$, respectivamente, no percentual de germinação. Tonetti et al. (2006) observaram resultados semelhantes ao analisar a qualidade física de sementes de E. erythropappus, representando um incremento na germinação de $76 \%$ após elas passarem pelo soprador de fluxo de ar.

Tabela 2. Percentual de germinação e de sementes vazias obtido pelo teste de germinação, e semente cheia obtido pelo teste de raios X, provenientes de cinco municípios do estado de Minas Gerais (MG), após tratamento com soprador.

Table 2. Porcentual germination and empty seeds obtained by the test of seed germination and full test obtained by x-ray, from five municipalities in the state of Minas Gerais, after treatment with blower.

\begin{tabular}{lccc}
\hline Procedência & Progênie & Germinação (\%) & Sementes vazias (\%) \\
\hline C25 & $95 \mathrm{a}$ & $1 \mathrm{a}$ & $99 \mathrm{a}$ \\
C6 & $92 \mathrm{a}$ & $0 \mathrm{a}$ & $100 \mathrm{a}$ \\
M22 & $86 \mathrm{ab}$ & $3 \mathrm{ab}$ & $96 \mathrm{a}$ \\
M24 & $84 \mathrm{ab}$ & $0 \mathrm{ab}$ & $100 \mathrm{a}$ \\
I18 & $84 \mathrm{ab}$ & $5 \mathrm{~b}$ & $95 \mathrm{a}$ \\
D2 & $83 \mathrm{ab}$ & $0 \mathrm{c}$ & $100 \mathrm{a}$ \\
D16 & $71 \mathrm{c}$ & $6 \mathrm{c}$ & $95 \mathrm{a}$ \\
B9 & $70 \mathrm{c}$ & $0 \mathrm{c}$ & $100 \mathrm{a}$ \\
B24 & $50 \mathrm{~d}$ & $48 \mathrm{~d}$ & $49 \mathrm{~b}$ \\
I12 & $12 \mathrm{e}$ & $82 \mathrm{e}$ & $14 \mathrm{c}$ \\
\hline
\end{tabular}

Médias seguidas de mesma letra não diferem entre si pelo teste de Tukey a 5\% de probabilidade.

Observou-se também que a progênie C25 foi a que apresentou menor percentual de germinação, sendo que, após o uso do soprador, ela igualou-se com a de maior destaque antes do tratamento (Tabela 2 e Figura 1).

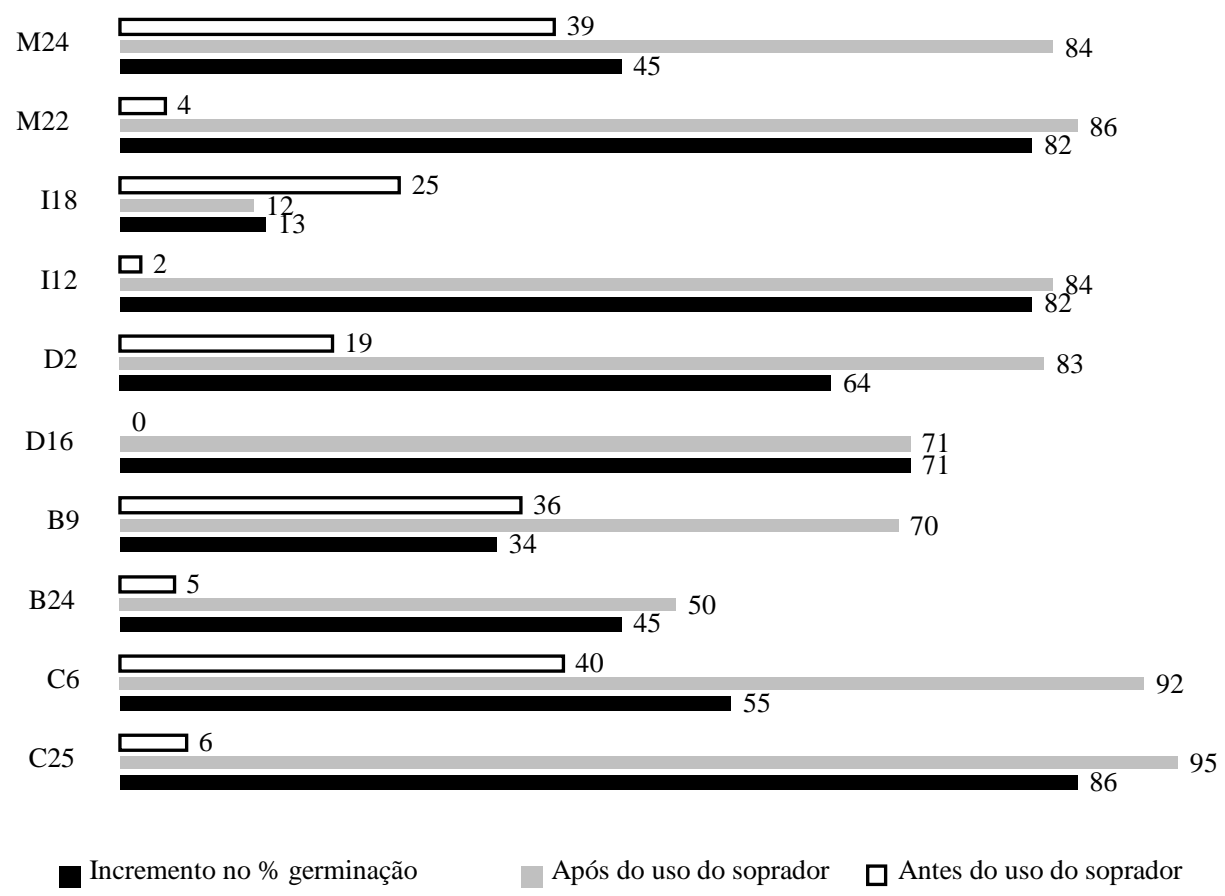

Figura 1. Percentual de germinação antes e após uso do soprador com os respectivos incrementos. Figure 1. Porcentual germination before and after using the blower with their increments. 
O incremento no percentual germinativo após a separação das sementes evidencia a necessidade do tratamento com soprador para a espécie. Algumas progênies estudadas atingiram valores acima de $80 \%$ pontos percentuais, o que pode ser considerado bom, em se tratando de espécies florestais. Portanto, recomenda-se a utilização de soprador de fluxo de ar para sementes dessa espécie, uma vez que elas apresentam grande número de sementes chochas.

\title{
CONCLUSÕES
}

- O baixo percentual germinativo de candeia (Eremanthus erythropappus) pode ser atribuído ao grande número de aquênio vazio e/ou embrião malformado.

- A progênie C6 foi a que melhor se destacou em todos os parâmetros analisados.

- O procedimento contribui significativamente no aumento do percentual de germinação.

- O uso da técnica de raios X é viável para auxiliar na avaliação da qualidade física de sementes de candeia (Eremanthus erythropappus).

\section{REFERÊNCIAS}

BARROSO, G. Compositae: Subtribo Baccharinidae Hoffmann. Estudo das espécies ocorrentes no Brasil. Rodriguésia, Rio de janeiro, v. 40, n. 2, p. 260-273, 1976.

CARPI, S. M. F.; BARBEDO, C. J.; MARCOS-FILHO, J. Condicionamento osmótico de sementes de Cedrela fissilis Vell. Revista Brasileira de Sementes, Brasília, DF, v. 18, n. 1, p. 271-275, 1996.

CARVALHO, P. E. R. Espécies florestais brasileiras: Recomendações silviculturais, potencialidade e uso da madeira. Brasília, DF,: EMBRAPA - CNPF, 1994. 640 p.

CARVALHO, N. M, Nakagwa. Sementes: ciência, tecnologia e produção. $4^{a}$ ed. Campinas: Fundação Cargill, 2000. $429 \mathrm{p}$.

CHAVES, M. M. F.; RAMALHO, R. da S. Estudos morfológicos em sementes, plântulas e mudas de duas arbóreas pioneiras da família Asteraceae (Vanillosmopsis erythropappa Sch. Bip e Vernonia discolor (Spreng.) Less). Revista Árvore, Viçosa, MG, v. 20, n. 1, p. 1-7. 1996.

DAVIDE, A. C.; TONETTI, O. A. O. Germinação de sementes de Erementhus erythropappus (Candeia) sob dois regimes de temperatura e luz. Informativo ABRATES, Londrina, v. 13, n. 1, set. 2003.

GOMES, V.; FERNANDES, W. Germinação de aquênios de Baccharis dracunculifolia D. C. (Asteraceae). Acta Botânica Brasílica, São Paulo, v. 16, n. 4, p. 421-427. 2002.

MACHADO, C. F.; CICERO, S. M. Aroeira-branca [Lithraea molleoides (Vell.) Engel.-Anacardiaceae] seed quality evaluation by the x- Ray test. Sciencia Agrícola, Piracicaba, v. 60, n. 2, p. 393-397, 2003.

\author{
NÚCLEO DE ESTUDOS EM MANEJO FLORESTAL (NEMAF). Sistema de manejo para a Candeia \\ (Eremanthus erythropappus e Eremanthus incanus). Disponível em: <http/l \\ www.nucleoestudo.ufla.br/nemaf>. Acesso em: 25/02/2008.
}

PEREIRA, T. S.; MANTOVANI, W. Maturação e dispersão de Miconia cinnamomifolia (DC) Naud. na reserva biológica de Poços das Antas, município de Silva Jardim, RJ, Brasil. Acta Botânica Brasilic,. São Paulo, v. 15, n. 3, p. 335-348. 2001.

PÉREZ, J. F. M. Sistema de manejo para candeia (Eremanthus erythropappus (DC.) MacLeish). 71 p. Dissertação de mestrado em Ciências Florestais - Universidade Federal de Lavras, Lavras, 2001.

SAlUSTIANO, M. E.; FERRAZ FILHO, A. C.; POZZA, E. A.; CASTRO, H. A. Extratos de candeia (Eremanthus erythropappus (DC.) MacLeish) na inibição in vitro de Cylindrocladium scoparium e de quatro espécies de ferrugens. Cerne, Lavras, v. 12, n. 2, p. 189-193, 2006. 
SCOLFORO, J. R. S; OLIVEIRA, A. D. de; DAVIDE, A. C.; MELLO, J. M. de; ACERBI JUNIOR, F. W. Manejo sustentável da candeia Eremanthus erythropappus e Eremanthus incanus: relatório técnico científico. Lavras: UFLA-FAEPE, 2002. 350 p.

SILVA, J. G. da; FONSECA, J. R. Colheita e pós-colheita. In: AIDAR, H. (Ed.). Cultivo do feijoeiro comum. Santo Antônio de Goiás: Embrapa Arroz e Feijão; Campinas: Embrapa Informática Agropecuária, 2003.

SILVA, J. G. da; FONSECA, J. R. Colheita mecanizada. In: AIDAR, H.; BIAVA, M. (Ed.). Produção de sementes sadias de feijão comum em várzeas tropicais. Santo Antônio de Goiás: Embrapa Arroz e Feijão; Campinas: Embrapa Informática Agropecuária, 2004. (Embrapa Arroz e Feijão. Sistemas de Produção, 4).

SOUZA, J. R. P.; AGUILERA, D. B.; TAKAHASHI, L. S. A. Germinação de sementes de camomila (Matricaria chamomilla L.) separadas com espalhante adesivo. Revista Brasileira de Sementes, Brasília, DF, v. 23, n. 1, p. 285-289, 2001.

TEIXEIRA, M. C. B.; NUNES, Y. R. F.; MAIA, K. M. P.; RIBEIRO, R. N. Influência da luz na germinação de sementes de candeia (Vanillosmopsis erythropappa Shuh. Bip.). In: ENCONTRO REGIONAL DE BOTÂNICA, 1996, Belo Horizonte. Anais... Belo Horizonte: SBB: Pontifícia Universidade Católica de Minas Gerais, 1996, p. 35-41.

TONETTI, O. A. O. Melhoria da qualidade física e estudo da germinação de sementes de candeia (Eremanthus incanus (Less) e Eremanthus erythropappus (DC.) Mac Leisch). 72 f. Dissertação (Mestrado em Ciências Florestais) - Universidade Federal de Lavras, Lavras, 2004.

TONETTI, O. A. O.; DAVIDE, A. C.; SILVA, E. A. A. Qualidade física e fisiológica de sementes de Eremanthus erythropappus (DC.) Mac.Leish. Revista Brasileira de Sementes, v. 28, n. 1, p.114-121, 2006.

VELTEN, S. B.; GARCIA, Q. S. Efeitos da luz e da temperatura na germinação de sementes de Eremanthus (Asteraceae), ocorrentes na Serra do Cipó, MG, Brasil. Acta Botânica Brasilica, São Paulo, v. 19 , n. 4 , p. $753-761.2005$. 